\title{
HACIA UNA FENOMENOLOGÍA DEL TIEMPO. UNA INTERPRETACIÓN DE LAS CRÍTICAS DE HUSSERL A BRENTANO EN LAS LECCIONES DE FENOMENOLOGÍA DE LA CONCIENCIA INTERNA DEL TIEMPO
}

\author{
TOWARD A PHENOMENOLOGY OF TIME. AN \\ INTERPRETATION OF HUSSERL'S CRITICISM TO \\ BRENTANO IN THE LECTURES ON INTERNAL \\ TIME CONSCIOUSNESS FROM THE YEAR 1905
}

\author{
VERÓNICA KRETSCHEL" \\ Universidad de Buenos Aires
}

\begin{abstract}
Resumen: La primera parte de las Lecciones de fenomenología de la conciencia interna del tiempo consiste en una exposición crítica de la teoría del tiempo del maestro de Edmund Husserl, Franz Brentano. Se ha señalado el carácter infundado de las críticas allí planteadas. Frente a esto, nuestra intención es enfatizar el carácter contextual de tales críticas. Esto significará buscar defender la idea de que la presentación de la teoría brentaniana no debe evaluarse de acuerdo a la justeza en la interpretación de su letra, sino como una base sobre la cual la fenomenología del tiempo husserliana se asentará. En particular, se desprenderán de estas críticas aspectos centrales de los que deberá dar cuenta la conciencia temporal: la originariedad del pasado y la temporalidad de los actos son algunos de los aspectos más relevantes. Nuestra propuesta buscará, según esto, explicitar la doctrina brentaniana del tiempo según la presentación de las Lecciones,
\end{abstract}

\footnotetext{
* Becaria Posdoctoral Conicet-Docente Universidad de Buenos Aires. Av. Caseros $9412^{\circ}$ D. CABA (1152). veronicakretschel@gmail.com
} 
analizar las críticas de Husserl y determinar las pautas allí subyacentes para una fenomenología del tiempo.

Palabras clave: Husserl, Brentano, Fenomenología del tiempo, Temporalidad.

Aвstract: The first part of The Lectures on Internal Time Consciousness from the Year 1905 consists of an explanation of the time theory of Edmund Husserl's teacher, Franz Brentano. The critical aspect of this presentation of Brentano has been shown to be not entirely valid. Given this consideration, our intention here is to emphasize the contextual character of these criticisms. This will involve defending the idea that they must not be evaluated by comparing the Husserlian interpretation with Brentanian theory, but by the motivation that this provides as the very beginning of the phenomenology of time. There are, in particular, some core issues which internal time consciousness in phenomenology should attend to and that were deduced from the Brentanian criticisms: the originality of the past and the temporalization of acts are some of the more relevant aspects. According to this, our proposal would try to specify Brentanian doctrine as it is presented in the Lectures, analyze Husserl's criticisms, and determine the underlying clues regarding a phenomenology of time.

KeYwORDs: Husserl, Brentano, phenomenology of time, temporality

\section{Introducción}

Las Lecciones de fenomenología de la conciencia interna del tiempo comienzan con una toma de posición metodológica en la cual Husserl delinea cuál será el objeto de estudio de la fenomenología del tiempo; esto es: la constitución inmanente del tiempo que parte de la duración como dato fenomenológico. A continuación se presentan una serie de parágrafos dedicados a la teoría del tiempo de Franz Brentano. Podría suponerse que el objeto de introducir esta teoría es que opere de referencia para los estudiantes de Husserl, como un horizonte a partir del cual poder entender su propia teoría. Esto tendría sentido en caso de que estuvieran familiarizados con la doctrina de Brentano. Sin embargo, la teoría del tiempo a la que se hace referencia no tuvo amplia difusión. No fue publicada por su autor, sino que fue presentada en el curso Ausgewählte psychologische und ästhetiche Fragen, al que Husserl asistió. Sus anotaciones ${ }^{1}$, junto con

${ }^{1}$ Estas anotaciones se encuentran en el Archico Husserl de Lovaina, catalogadas como Q 9. Existe también una reseńa sobre el curso en Hua XXV, 307. 
la de otros asistentes, son el único testimonio publicado de la teoría presentada ${ }^{2}$. A su vez, tampoco parece factible afirmar que las tesis allí expuestas expresen la posición definitiva de Brentano sobre el tema. En efecto, en 1905 ya habrían sido abandonadas las tesis descriptas por Husserl (Brentano, 1995, 175-177, nota 6$)^{3}$, acercándose, aparentemente, a una interpretación más parecida a la de su discípulo (de Warren, 2009, 55, nota 15).

De modo póstumo se han publicado escritos de Brentano en los que se encuentran, ahí sí de modo directo, descripciones en torno al tema del tiempo que sirven para complementar lo expuesto por Husserl en estos primeros parágrafos de las Lecciones. Es el caso de Deskriptive Psychologie y de las Philosophische Untersuchungen zu Raum, Zeit und Kontinuum. El primer texto recoge lecciones impartidas por Brentano en la Universidad de Viena entre 1887 y 1901 . Si bien Husserl por esa época ya no estaba presente en los cursos de Viena, sí contaba con una transcripción de uno de ellos (el de 1887/1888, llamado también "Psicología descriptiva"). En ese sentido, podemos sostener que las fuentes para su crítica en las Lecciones serían la mencionada transcripción y sus notas del curso Ausgewählte psychologische und ästhetiche Fragen. Lo cual indica que no buscó a la hora de preparar su texto conocer en qué estado se encontraba la teoría del tiempo de su maestro, o si había habido alguna modificación en relación con el contenido de sus notas. Por un lado, esto ocasionó que las críticas no fueran del todo justas. Pero, por otro lado, el mismo hecho es prueba del interés que la antigua teoría brentaniana sobre el tiempo provocaba en Husserl. En este contexto, es interesante preguntarse en qué se funda tal interés. Es decir, independientemente de las críticas, esta teoría es elegida como punto de partida para el desarrollo de la fenomenología del tiempo. Nuestro objetivo en lo siguiente será intentar ahondar en esta cuestión. Esto implicará, por una parte, exponer la doctrina brentaniana del tiempo y, por otra, reconstruir y evaluar las críticas que Husserl le dirige.

\footnotetext{
${ }^{2}$ Anton Marty en su Die Frage nach der geschichtlichen Entwicklung der Farbensinnes y Carl Stumpf en su Tonpsychologie

${ }^{3}$ Aquí los editores reconstruyen las modificaciones posteriores que introdujo Brentano en su teoría del tiempo, haciendo referencia a algunas de las objeciones que suelen planteársele.
} 


\section{Brentano y la asociación originaria}

Según Nicolas de Warren, la teoría brentaniana en el estado de avance en que la presenta su discípulo se instala entre dos referentes de la historia del concepto de tiempo: Aristóteles y Locke. Pese a la diferencia radical en el modo de concebir el tiempo entre estos autores, ambos comparten algo: sus explicaciones no permiten explicar la experiencia subjetiva del tiempo. De las críticas que Brentano les dirige se desprenden algunas características que poseerá su propia teoría del tiempo: 1. el tiempo será estudiado como un fenómeno subjetivo; 2 . su teoría deberá permitir "percibir" el pasado junto con el ahora; 3. la sucesión de la conciencia presupondrá la conciencia de la sucesión; 4. el pasado significará una modificación respecto al presente. Dicho esto, comencemos por reconstruir la teoría de Brentano tal como es presentada en las Lecciones. Cuando lo que afirme Husserl así lo requiera lo complementaremos a partir de los textos del autor.

A través de la exposición de la doctrina de su maestro Husserl pretende "ganar un acceso a los problemas planteados" (Husserl, 2002, 33). Busca destacar, por tanto, dónde radica la novedad, lo que consistirá en gran medida en establecer cuál es la posición de Brentano respecto de las teorías psicológicas sobre el origen del tiempo. Esto implicará dar respuesta a preguntas del tipo: ¿es posible encontrar en esta teoría algo diferente de una respuesta psicológica acerca del origen del tiempo? ¿es completamente superadora la tesis de Brentano de aquellas que se fundan sobre bases empíricas? Recordemos que, si bien ya en los "Prolegómenos" a las Investigaciones Lógicas las críticas al psicologismo habían sido efectuadas, no había sido en ese caso Brentano objeto de cuestionamiento. El propio Husserl le asegura en una carta de 1905 que no hay nada en los "Prolegómenos" en contra suyo (Mohanty, 2011, 69). Con todo, no dudará aquí, texto datado en el mismo año, en atribuirle una tesis psicologista.

La objeción central que le dirige Husserl al psicologismo es que conduciría inexorablemente o a un reduccionismo, o a algún tipo de relativismo. Estas consecuencias parecen no estar presentes en la filosofía de Brentano y podemos presumir que tendría ese sentido la afirmación de 1905. Respecto de las teorías acerca del origen del tiempo, la psicología, sea innatista o empirista, se ha concentrado en explicar la intuición objetiva del tiempo a partir del material originario de la sensación. Pero, esto no nos concierne a nosotros, fenómenologos, afirma Husserl, dado que es una mera "génesis empírica" del fenómeno del tiempo (Husserl, 2002, 31). La pregunta fenomenológica no puede tener una 
respuesta empírica, en la medida en que los datos de la experiencia han quedado excluidos por medio de la suspensión de la tesis de existencia de toda trascendencia. La fenomenología busca, por el contrario: "hacer claridad sobre el a priori del tiempo, explorando la conciencia del tiempo, sacando a la luz su constitución esencial y poniendo de manifiesto los contenidos de aprehensión y los caracteres de acto específicos, en su caso, del tiempo, a los que pertenecen por esencia las leyes aprióricas del tiempo" (Husserl, 2002, 31). A partir de esta frase se puede determinar en qué consiste el estudio fenomenológico del tiempo: un análisis de las vivencias en sentido puro, sean éstas intencionales (caracteres de acto) o no-intencionales (contenidos de aprehensión). Pero otro ha sido el proceder de la psicología. Al reconducir el origen del tiempo al aspecto empírico del material sensible, ha asumido el carácter objetivo de la temporalidad, pretendiendo, con ello, dar cuenta del aparecer subjetivo en base al aspecto temporal del dato sensible. Este aspecto originaría, según estas concepciones, nuestra intuición de tiempo. En este contexto, la posición de Brentano marca una diferencia: su intención es describir nuestra experiencia del tiempo, sin asumir, en primera instancia, la existencia de un tiempo objetivo.

Para fortalecer la comprensión de la doctrina brentaniana del tiempo, resulta necesario relacionarla con su proyecto filosófico -la psicología descriptiva-, marcando, sobre todo, las diferencias con la psicología criticada. En este punto, será posible observar una notable coincidencia entre Brentano y Husserl; vale la pena mencionar que el propio Husserl define la fenomenología de las Investigaciones Lógicas como psicología descriptiva. Brentano distingue entre la psicología descriptiva (o psicognosia) y la psicología genética. Mientras que la primera "apunta a determinar exhaustivamente, si es posible, los elementos de la conciencia humana y los modos según los cuales estos se conectan" (Brentano, 1995, 3); la segunda procura "describir las condiciones causales a las cuales los fenómenos individuales están sujetos" (Brentano, 1995, 3). Estas diferencias respecto a sus objetivos generan, a su vez, que las leyes de cada ciencia no coincidan respecto a su status (Mulligan y Smith, 1985, 629). La psicología descriptiva alcanza leyes exactas y universales, que son inmediatas, autoevidentes y apodícticas. Esto les otorga un status privilegiado, de leyes fundamentales, al que hay que agregar el carácter fundante que poseen respecto a las leyes de otras ciencias. En contrapartida, las leyes de la psicología genética son inexactas, faltas de evidencia y tienen un carácter meramente presumptivo. Luego, las últimas se encuentran fundadas en las primeras. Como afirma Brentano: "La psicognosia es prioritaria en el orden natural" (Brentano, 1995, 8). Si bien pueden darse casos en los que la psicología decriptiva emplee recursos de la psicología genética (Brentano, 1995, 8-10), no 
hay duda acerca de la prioridad de la primera. En ese sentido, los estudios de un psicólogo genético que no tenga en cuenta los fundamentos de la psicología descriptiva son considerados vanos (Brentano, 1995, 10).

El método de la psicología descriptiva tiene como presupuesto la percepción interna. Esto es: el hecho de que tenemos una conciencia presente de cada uno de nuestros actos. En efecto, cada acto apunta siempre en dirección a dos objetos. Por una parte, se dirige hacia su objeto primario: el objeto en sentido estricto, el objeto de la percepción externa; es decir: fenómenos físicos percibidos a través de los órganos de los sentidos, como son, por ejemplo, las cualidades sensibles

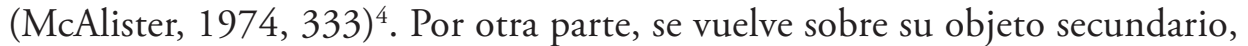
que no es otra cosa que el propio acto. Esto significa que todo acto implica un "notar" (bemerken) el acto y a esto lo denominamos objeto secundario del acto. Este advertir que tiene lugar toda vez que tengo una percepción sensible, por referirnos a un tipo básico de acto, es en sí mismo autoevidente. Igual que en Husserl, tenemos en Brentano una evidencia absoluta de nuestra autocaptación. Con todo, algo importante para destacar -y aquí sí habrá una diferencia radical con las tesis husserlianas- es que esta conciencia es una conciencia presente. $\mathrm{O}$ sea, sólo tenemos conciencia de un acto mientras este acto se está dando. En este sentido, la teoría del tiempo de Brentano deberá explicar qué es lo que ocurre respecto a la conciencia pasada de un acto. Esto implica responder si y -en caso afirmativo- cómo tengo conciencia actual de un acto pasado.

Un lugar común de las teorías del tiempo es explicar la temporalidad en términos de sucesión de ideas en la conciencia. Entonces, si explicamos cómo se produce la sucesión en la conciencia, habremos explicado el origen de lo temporal. Estas teorías parecen enfrentarse a un círculo vicioso cuando pretenden determinar si la sucesión de conciencia es o no prioritaria respecto a la conciencia de la sucesión. Anticipamos que Brentano se inclina por afirmar que no es posible pensar la sucesión sin la conciencia de sucesión. Es así que en la reconstrucción de su doctrina presente en las Lecciones se reconduce la cuestión de la sucesión temporal al problema de nuestra experiencia de dicha sucesión. Para poder experimentar una sucesión de sensaciones es necesario que algo más que una concatenación de sensaciones se suceda. Por ejemplo: para que sea posible escuchar una melodía no sólo es necesario que se den una serie de notas, sino

\footnotetext{
${ }^{4}$ La autora explica aquí por qué un fenómeno físico debe ser entendido como una cualidad sensible en Brentano, en discusión con los ejemplos de Chisholm, para quien los fenómenos físicos son actividades.
} 
que sean experimentadas en la forma de una sucesión. Si esa conexión entre las notas no se diera, no escucharíamos una melodía sino meramente unas notas yuxtapuestas. Lo que busca es, por tanto, explicar cómo se produce la conciencia de la sucesión, qué opera la conexión entre las notas. Según esto, es necesario una operación que conecte las distintas sensaciones y que dé cuenta de nuestra experiencia de la sucesión. A la reunión de las sensaciones Brentano la llama asociación originaria o proteraesthesis.

El concepto de asociación originaria no debe ser rastreado en la historia de la filosofía. Es una noción introducida por Brentano para dar cuenta del origen del tiempo y, como él mismo señala, es muy difícil de explicar:

"Cuando hablé de contenido de experiencia, todos ustedes, presumiblemente, entendieron más o menos qué quise decir; ahora, que hablo de asociación originaria, debo temer que ninguno en mi audiencia sepa a qué estoy efectivamente apuntando. De hecho, la expresión no aparece en ningún manual o libro de texto de psicología. Y, me parece que el hecho al cual ella refiere no ha sido él mismo ni aprehendido ni interpretado en nada que yo haya visto. Yo mismo nunca he publicado nada sobre él, lo que explica por qué esta doctrina ha sido sólo enseñada oralmente en ciertas cátedras de discípulos míos" (R. Chisholm, W. Baumgartner y B. Müller, 1995, xxiii).

La idea es que cada vez que percibimos una sensación que dura (por ejemplo, un tono de una melodía que se distiende en el tiempo), tendemos a pensar que es la misma sensación la que dura. Lo que ocurre, sin embargo, es que lo que percibimos no es sólo una sensación presente, sino la sensación presente asociada con sensaciones pasadas; todas de una duración tan breve que es prácticamente imposible distinguirlas. Esa experiencia de duración es el resultado de la proteraesthesis: la asociación entre una sensación que está presente con otras que acaban de estarlo. Ahora bien, mientras que el fenómeno que explica la asociación originaria resulta tangible, no ocurre lo mismo con la noción de asociación originaria. Al dar cuenta del fenómeno de la duración, decimos que una sensación se asocia con sensaciones pasadas. ¿Esto significa, entonces, que la proteraesthesis es una sensación? ¿O que ella, como acto, tiene como objeto una sensación pasada? 
Ni lo uno, ni lo otro. La proteraesthesis es un acto que tiene como objeto el objeto secundario de la percepción sensible (Brentano, 1982, 98).

Para Brentano todos los actos apuntan en dos direcciones. La percepción sensible, por tanto, tiene dos objetos. Un objeto primario que es la cualidad sensible (la nota de la melodía) y un objeto secundario que es la percepción misma de esa cualidad (el sentir la nota). La proteraesthesis, por su parte, tiene también dos objetos. La diferencia es que el objeto primario no es trascendente, sino inmanente; el objeto primario de la proteraesthesis es el sentir del sonido pasado, o sobreinterpretando un poco a Brentano, el haber recién sentido un sonido. Finalmente, deberíamos asumir que el objeto secundario sería la autoconciencia de la experiencia pasada del sonido. Frente a la dificultad de este concepto de Warren propone encarar la interpretación de "proteraesthesis" como: "un desafío específico: se nos pide considerar la posibilidad de una percepción sensible que no es ni memoria, ni percepción, la cual revela el pasado inmediato junto con el presente. Se nos pide, en otras palabras, percibir lo recién pasado o 'el antes que' (protero-asthesis)" (de Warren, 2009, 60). Y la tarea que se nos pide no es menor, sobre todo teniendo en cuenta que percibir lo recién pasado es percibir algo que ya no existe. ¿Puede salvar este problema pensar que lo que "percibimos" en la proteraesthesis es un acto (el sentir pasado) y no una sensación? No parece tan sencillo de asumir, en la medida en que lo que hace que la sensación pasada sea no-existente es su condición de pasada que comparte con el acto de sentir. Dejando de lado estas dificultades, retomemos la descripción del origen del concepto de tiempo, tal como la plantea Husserl.

Afirma Husserl: "Cuando vemos algo, oímos algo, o en general lo percibimos, sucede de manera regular que lo percibido permanece presente para nosotros un lapso de tiempo, pero no sin modificarse" (Husserl, 2002, 33). Esto se da porque, por ejemplo, cuando percibimos una melodía una nota está presente y las notas pasadas se hacen presentes, de modo modificado. A cada nueva sensación se le une una serie continua de sensaciones "peculiarmente modificadas" (Husserl, 2002, 34). Y la proteraesthesis es el acto que intenciona esas "sensaciones pasadas" o, mejor dicho, ese haber experienciado tales sensaciones. Ahora bien, dos funciones deben producirse para que tengamos conciencia de la sucesión. Por un lado, obviamente, la asociación de las sensaciones. Por otro, y no menos importante, una modificación constante. Si lo que queremos describir es el fenómeno del tiempo a través de la conciencia de la sucesión, el pasaje del tiempo del presente al pasado, deberá ser un foco central de nuestra investigación. En efecto, esa "peculiar modificación" que opera en las sensaciones cuando dejan de estar 
presentes es lo que genera nuestra conciencia de pasado. En palabras de Husserl: "Llega primeramente a alcanzarse la representación de la sucesión gracias a que la sensación anterior no persiste invariada en la conciencia, sino que se modifica de modo peculiar, y justamente se modifica sin cesar de momento en momento" (Husserl, 2002, 35). La asociación originaria "reproduce" o, mejor, "presenta" el contenido de la sensación original y, con ello, el momento de tiempo que a ella le correspondía. Se sigue de esto, para Husserl, que las sensaciones, están compuestas por una materia sensible y por un contenido temporal.

Vale la pena tener en cuenta aquí el análisis que lleva a cabo Brentano en Psicología descriptiva, donde busca, justamente, determinar cuáles son los actos fundamentales y cuáles sus objetos primarios (Brentano, 1982, 92-98). En efecto todo acto fundamental posee un objeto primario (p. e.: un fenómeno sensorial) que tiene, a su vez, al menos dos partes: determinación espacial y cualidad. En este contexto se pregunta si el tiempo no será otro componente de los actos. La estrategia para responder esta pregunta es determinar si es posible atribuirle un carácter temporal a la sensación. Entonces, mientras que afirma que ya sabemos acerca del carácter temporal de la proteraesthesis, se cuestiona si es posible que la sensación posea también determinación temporal. No obstante esto: ¿cómo sabemos, en efecto, del carácter temporal de la proteraesthesis? Como ella es "sensación pasada”, sostiene Brentano, su definición implica su temporalidad. ¿Y qué significa que sea temporal? Que es una modificación; ser pasado es haber sufrido una modificación. Según esto: ¿̨ocurre lo mismo con la sensación? En principio, deberíamos decir que no, dado que no comporta ninguna modificación. De hecho, ser presente implica no estar modificado. Sin embargo, se pregunta Brentano, si no podría pensarse el presente en relación con la modificación; es decir: en relación con el tiempo. El presente podría ser concebido como el punto cero de la modificación ${ }^{5}$, como el comienzo de la temporalidad. Estos argumentos no terminan de convencer al propio Brentano, con lo cual deja abierta la cuestión de si la sensación es temporal. Lo que confirma, no obstante, es el carácter indudablemente temporal de la proteraesthesis.

En este marco, resta determinar si es válida la afirmación de Husserl acerca del carácter temporal de la sensación. Esta cuestión puede ser abordada desde varias perspectivas. Por una parte, si entendemos que Husserl considera la

\footnotetext{
${ }^{5}$ Interesante sería comparar aquí la propuesta de Brentano de considerar la sensación presente como punto cero de la modificación, en relación con la caracterización husserliana del ahora como límite.
} 
sensación en sentido estricto - i. e.: como sensación presente- su crítica es ilégitima. No parece, no obstante, ser este el caso, como veremos en su descripción. Por otra parte, si se refiere a las sensaciones efectivamente temporales, si está limitando su estudio a las sensaciones de pasado, podría su presentación ser válida. Esta última interpretación tiene, con todo, inconvenientes que analizaremos más adelante.

Volviendo a la descripción husserliana, las sensaciones permanecerían ordenadas fuera del campo intuitivo en base al aspecto temporal que permanece invariado con el retroceso al pasado. Esta reproducción del contenido de las sensaciones que pasaron (o asociación originaria), afirma Husserl, es resultado de la fantasía. En efecto, es la fantasía aquella operación capaz de producir la presentación de la sensación pasada; asiste a la percepción, al extender de modo productivo el presente intuitivo hacia lo ya no intuitivo. Con todo, es importante destacar que es la fantasía productiva y no la rememoración la que produce la asociación originaria. Su característica esencial es mantener en la presencia algo no intuitivo, no recordar o hacer intuitivo algo que fue presente. Así, mientras que la rememoración es una presentación inauténtica, dado que reproduce un darse originario; la fantasía, a causa de su carácter productivo, es una presentación irreal (de Warren, 2009, 85). En suma: nuestra noción de tiempo requiere de la experiencia de la sucesión y esta experiencia está fundada en la asociación originaria, producto de la fantasía. De este modo, en la medida en que la fantasía produce presentaciones irreales, nuestra noción de tiempo es irreal. Sostiene Husserl, luego, que: “...Brentano viene a negar la percepción de la sucesión y del cambio” (Husserl, 2002, 36).

Retomando algunas cuestiones que habían quedado abiertas más arriba, ¿cómo puede la proteraesthesis intencionar algo pasado, algo no-existente? La observación de Husserl parece adecuada: produciendo de modo fantasioso algo que ya no está. Y, como corolario, si sólo podemos tener experiencia real de aquello que se da sin intervención de la asociación originaria, no sólo nuestro concepto de tiempo es irreal, sino que tampoco es posible atribuirle realidad a nuestro pasado. Lo que resta analizar, no obstante, es en qué medida pueden equipararse las distintas maneras de caracterizar el pasado que aquí tratamos: ¿es lo mismo sostener que nuestra percepción de la sucesión está montada sobre una producción de la fantasía y que tal experiencia es una ilusión? ¿todas las creaciones de la fantasía poseen un carácter irreal? Claro que no. Mientras que, en sentido estricto, la fantasía -y así lo hará Husserl en los textos de finales de la década de '1910- se entiende como un acto que intenciona un objeto que 
no tiene posición temporal, diríamos, que no existe efectivamente; en sentido amplio puede entenderse como un acto de representación, más asociado a la memoria. Ya dijimos porqué esta fantasía no es la memoria, sin embargo, no parece que pueda igualarse, tampoco, al sentido estricto de fantasía. Con todo, deberemos quedarnos, al menos por ahora, con la posición de Husserl al respecto, a fin de desarrollar con mayor precisión en qué consisten sus críticas a Brentano y en qué medida son relevantes para su propia teoría.

\section{Las críticas de Husserl}

La irrealidad del tiempo y la existencia del pasado han sido temas ampliamente abordadas por la tradición filosófica. No fue Husserl, tampoco, la excepción. El hecho de que nuestra experiencia de sucesión se dé a partir de presentaciones producto de la fantasía y, en consecuencia, irreales, parece conducir a la idea de que el tiempo en sí mismo es irreal. Nosotros "creemos oír una melodía, creemos oír aún, por tanto, también lo que acaba de pasar, mas ello es sólo una ilusión" (Husserl, 2002, 36), afirma Husserl, poniéndose en la voz de Brentano, como un modo de anticipar una de las consecuencias más fuertes de la teoría que inmediatamente criticará. Porque: jen qué medida podemos sostener el carácter ilusorio de una experiencia tan cotidiana como nuestra experiencia del tiempo? Comenzamos planteando que Brentano había dado un paso más allá de las teorías psicologistas: buscar el origen del tiempo en condiciones subjetivas. Ahora bien: ¿que la experiencia de tiempo sea producto de la fantasía y, con ello, irreal no termina neutralizando el gesto antipsicologista de Brentano?

En Brentano ve Husserl no sólo un gesto antireduccionista (Husserl, 2002, 41), sino también un núcleo fenomenológico (Husserl, 2002, 38). Por una parte, no busca explicar el tiempo a partir de una representación inmanente del tiempo objetivo. Por otra, reconduce el problema al fenómeno que se nos manifiesta: la experiencia de la sucesión. Pese a esto, hay algo que falla: no logra dar cuenta del fenómeno que pretende describir. El tiempo no es una ilusión y el pasado tiene una dignidad en sí mismo que es necesario reconocer y explicitar. El error de Brentano radica, entiende Husserl, en no reconocer los elementos propios del fenómeno que intentó analizar. Si bien había sido él el primero en deslindar los contenidos primarios de los caracteres de acto, a la hora de estudiar el tiempo terminó asignando temporalidad a un aspecto de la sensación. El dato sensible, al menos en la forma de proteraesthesis, posee un momento temporal. En este 
sentido, nuestra experiencia de tiempo no se describe puramente en la inmanencia de las operaciones subjetivas, sino que presupone un dato externo que ya es temporal. Esta necesidad de asumir un aspecto temporal -trascendente- de la sensación implicaría una posición psicologista. Con todo, cabe sopesar aquí también las rotundas afirmaciones de Husserl. Porque: ¿si la proteraesthesis tiene como objeto una experiencia pasada, en qué medida podemos decir que el tiempo proviene de una instancia trascendente? Hay que reconocer que en todo esto hay bastante de especulación, pero: ¿no afirma Brentano que la proteraesthesis no tiene el mismo tipo de objeto que la percepción sensible? Y, en ese sentido: ¿no se apura Husserl en atribuirle una posición psicologista, a quien, tanto como él, pretendió establecer una ciencia pura que sea fundamento de la génesis empírica? Otra vez un problema que queda irresuelto, aunque será importante tenerlo en cuenta a la hora de establecer las consideraciones finales respecto a la influencia de esta teoría del tiempo en la del Husserl de 1905.

Ahora bien, hasta el momento, las críticas a la doctrina de Brentano apuntan, en dos direcciones. En primer lugar, hacia el rol de la proteraesthesis y las consecuencias psicologistas que éste implicaría. En segundo lugar, hacia el carácter productivo de la fantasía y el concepto irreal de pasado resultante. Estas dos vertientes de la crítica se anudan en un problema común. Afirma Husserl que, en definitiva, la deficiencia fundamental del análisis de Brentano radica en la imposibilidad de estudiar la constitución del tiempo, tomando en consideración "los caracteres de acto que son decisivos para tal teoría" (Husserl, 2002, 41). En función de esta deficiencia podemos identificar las siguientes objeciones puntuales:

1. Desde la teoría de Brentano sería imposible distinguir entre la percepción de una duración actual y el recuerdo de un trecho pasado. Si la fantasía opera tanto en la conformación de la duración presente, como en la presentificación (Vergegenwärtigung) de un trecho de tiempo que fue experienciado, no se explica cómo es posible distinguir entre la percepción de una duración y el recuerdo. Habría que recurrir a "fantasías de fantasías" (Husserl, 2002, 39) para dar cuenta del recuerdo, lo que redunda en una complicación adicional de la teoría.

2. Si bien la interpretación husserliana de la proteraesthesis no deja de generar controversias, es cierto que tampoco Brentano es definitivo respecto a su caracterización. Husserl le reclama no haber extendido el estudio del tiempo a todos los aspectos de la relación intencional. En efecto, Brentano no se expide acerca 
de la duración de los actos, sino meramente al fenómeno de asociación entre las sensaciones. En este sentido, el carácter temporal es atribuido a un solo aspecto de la relación intencional, i. e.: a la sensación, y no a los objetos ni a los actos. Mientras que, como señala Husserl: "el análisis ha de recorrer más bien todos los niveles de la constitución” (Husserl, 2002, 39).

3. Si decimos que cuando percibimos algo que dura en el presente, en realidad estamos percibiendo sensaciones de pasado asociadas: ¿no supone eso que las sensaciones de pasado son también presentes? Es decir: al afirmar que la intuición de tiempo -presente- implica la asociación originaria, sostendríamos que lo pasado está también presente: "Lo cual es un patente contrasentido" (Husserl, 2002, 40).

4. Y, como consecuencia de 3., si no tenemos el momento pasado de la sensación -que ahora está presente- de algún modo presente: ¿cómo sabemos que ese momento ha pasado? ¿cómo establecemos una diferencia entre la sensación presente y la pasada? Y, por tanto: “¿De dónde tenemos nosotros la idea de pasado?" (Husserl, 2002, 40).

5. El momento pasado que se le ańade a la sensación comporta la producción del pasado. A su vez, implica que esa sensación sea ahora "irreal". Lo que no explica es cómo un momento psíquico que se añade es capaz de "producir la irrealidad". En términos generales, la caracterización del pasado como noingrediente o no-existente es dudosa (Husserl, 2002, 41).

Ahora bien, estas objeciones ofrecen un apoyo fuerte a la interpretación de Husserl, según la cual la doctrina brentaniana del tiempo no puede dar cuenta de modo conclusivo de nuestra experiencia del tiempo. A su vez, estas críticas imponen un desafío importante a la propuesta husserliana, en el sentido de que plantea dificultades que su teoría debería resolver.

En las críticas a Brentano el problema de la irrealidad del pasado aparece como una cuestión crucial, sostenido en la idea de que el origen del concepto es la asociación originaria y en que la temporalidad tiene que ver sólo con una determinación de las sensaciones pasadas. Ahora bien, una tesis más profunda anida en la teoría de Brentano: "el dogma de la momentariedad de un todo 
en la conciencia" ${ }^{3}$, que está presupuesta en las objeciones recién planteadas. El dogma de la momentariedad consiste en lo siguiente: "en orden a la captación de una sucesión de representaciones ( $a y b$ por ejemplo), es necesario que ellas sean los objetos enteramente simultáneos de un saber relacional que, siendo por completo irreversible, las reúna en un acto único e indivisible" (Husserl, 2002, 43). Es decir que para tener conciencia de una sucesión se requiere de un acto de conciencia que relacione las representaciones involucradas. Junto con esto se encuentra el supuesto de que la percepción de un discurrir temporal tiene lugar en un ahora momentáneo, puntual e indivisible (Husserl, 2002, 43). Entonces, para tener conciencia de un todo sucesivo, todas las partes que lo integran tienen que coincidir en un ahora a través del acto que las reúne. Esto es: si tenemos la secuencia de las sensaciones A, B y C, necesitamos, para percibirla qua sucesión, que una conciencia momentánea agrupe al acto que intenciona $\mathrm{A}$, junto con el acto que percibe $\mathrm{B}$ y el acto que espera $\mathrm{C}$. Lo que esto pone de manifiesto es la crítica central de Husserl a Brentano: no poner en consideración la temporalidad -la duración- de los actos. En contraposición la fenomenología del tiempo procurará asegurar un esquema de la conciencia temporal que dé cuenta de la síntesis "sin más" de las distintas instancias temporales y que abarque todas las direcciones de la descripción.

\section{Conclusiones}

Hemos buscado establecer una posición de equilibrio entre dos aspectos. Por un lado, el valor que encuentra Husserl en los desarrollos de su maestro. Por otro lado, la rapidez con la que, en algunos casos, lo critica. Tres cuestiones son centrales a la hora de realizar un balance de esta relación. En primer lugar, el problema de la proteraesthesis. En segundo lugar, la necesidad de la fantasía. En tercer y último lugar, la prioridad del presente. Respecto al primer punto, hay que admitir que Husserl no lleva a cabo un análisis muy detallado de la asociación originaria. Eso deriva en la conclusión de que el dato temporal es externo y, con ello, en el psicologismo atribuido a Brentano. Si bien aquí la crítica no sería muy justa, sí resulta crucial el hecho de que el estudio del tiempo limitado al fenómeno de la proteraesthesis deja de lado la temporalidad de los otros aspectos de la relación intencional. En este caso, Husserl es muy preciso cuando señala que

\footnotetext{
${ }^{6}$ La noción, como lo asegura Husserl, fue introducida por William Stern en su "Physische Präsenzzeit" publicado en el Zeitschrift für Psychologie XIII en 1897.
} 
la investigación del fenómeno del tiempo debe abarcar todos los componentes de la conciencia. La segunda cuestión nos parece muy elocuente, sobre todo desde un punto de vista propositivo. Lo que tenemos que evaluar aquí no sería tanto la pertinencia de la crítica, como la toma de posición que lleva a cabo Husserl. Él pretende que el tiempo no sea producto de la fantasía por que está convencido de que eso le quita dignidad al fenómeno. En ese sentido, procurará que en su teoría todas las dimensiones de la temporalidad tengan un carácter originario. Finalmente, en relación con la tercera cuestión, se abre toda una dimensión de la interpretación de la teoría husserliana del tiempo en la que no profundizaremos aquí y que apunta a la pregunta acerca del carácter presencialista o no de la fenomenología del tiempo husserliana.

Ahora bien, llegado este punto parece atinado retomar el problema con el que nos enfrentamos al comenzar este trabajo. Husserl introduce su fenomenología del tiempo a partir de la exposición de la teoría de Brentano. Las tesis del maestro conjugan dos interpretaciones contrapuestas. Mientras que, por una parte, pretenden establecer un objeto de estudio desde una perspectiva que podríamos llamar fenomenológica, i. e.: al intentar dar cuenta de la experiencia del tiempo; por otra parte, el resultado al que llega en la descripción del fenómeno no parece ser correcta: la experiencia del tiempo resulta una ilusión producto de la fantasía y, con ello, el pasado carece de realidad. Esta presentación de la posición de Brentano le permite a Husserl posicionarse tanto desde un punto de vista metodológico como ontológico. Esto es: la teoría del conocimiento que propone establece un estudio de la inmanencia que deberá dar cuenta de nuestra experiencia del tiempo. En la medida que esta experiencia, en términos de duración, es el dato fenomenológico y punto de partida de la investigación, no puede devenir irreal en la descripción. Así: "La unidad de la conciencia que abarca intencionalmente lo presente y lo pasado es un dato fenomenológico. La cuestión es entonces si en realidad, como afirma Brentano, lo pasado aparece a esta conciencia en la forma de la fantasía" (Husserl, 2002, 38). Y la respuesta a esta pregunta será negativa: la filosofía husserliana debe permitir dar cuenta de la temporalidad y, a su vez, atribuir un carácter originario a la donación del pasado.

La fenomenología del tiempo tal cual es planteada en las Lecciones opera una desconexión del tiempo objetivo y reconduce el estudio de la temporalidad a la duración. Sería interesante confrontar eso con la tesis de Mohanty quien señala que la ruptura definitiva de Husserl con el círculo brentaniano se produce con el descubrimiento de la epojé (Mohanty, 2008, 267). En este sentido, la epojé le proporciona a Husserl el marco teórico necesario para abordar la cuestión del 
tiempo desde un punto de vista fenomenológico. Brentano, por el contrario, no habría dado con la metodología adecuada. Es decir, si bien su punto de partida y sus objetivos serían propios de la fenomenología, las falencias de su investigación mostrarían que permanece todavía en una actitud psicologista. Por su parte, Husserl considera -vía epojé- que la duración en tanto fenómeno inmanente es el único dato fenomenológico en sentido propio. Dar cuenta del tiempo es, entonces, dar cuenta de la duración y esto consiste no sólo en un estudio de la duración de la sensación, sino también en un análisis de los actos que, en tanto tales también duran. La duración de los objetos proviene, luego, de la duración de los actos. El estudio husserliano del tiempo se configura, entonces, como una caracterización de los actos y del modo en que éstos se temporalizan: i. e.: si los actos son temporales, debemos encontrar una estructura que los temporalice. Así como los actos le dan tiempo a los objetos, es la conciencia absoluta, ella misma por fuera del tiempo, aquello que posibilita la temporalidad de los actos.

Ahora bien, que la estructura que fundamenta la temporalidad tenga la forma de la conciencia absoluta, no es algo que vaya de suyo. Husserl alcanza una teoría del tiempo consistente con las críticas a Brentano recién, al menos, en 1908. Hacia 1905, coinciden varios intérpretes, no se entiende bien cómo su propia teoría podría resolver las objeciones realizadas al maestro (Gallagher, 1998, 43; de Warren, 2009, 143). Para concluir nos gustaría insistir con lo que nosotros creemos debe ser la manera de abordar las críticas a Brentano. En ellas están implícitas las cuestiones centrales que buscará responder Husserl. En ese sentido, más que una crítica en sentido estricto, los parágrafos estudiados deben ser pensados como la formulación de una propuesta.

\section{Bibliografía}

Brentano, Franz (1982). Deskriptive Psychologie. Hamburg: Meiner. (1995). Descriptive Psychologie. Londres: Routledge. (1976). Philosophische Untersuchungen zu Raum, Zeit und Kontinuum. Hamburg: Meiner.

Chisholm, Roderick, Baumgartner, Wilhelm y Müller, Benito (1995). "Introduction". Descriptive Psychology. Londres: Routledge.

de Warren, Nicolas (2009). Husserl and the Promise of Time. Cambridge: Cambridge University Press. 
Husserr, Edmiund (1986). Aufsätze und Vorträge (1911-1921) Mit ergänzenden Texten. La Haya: Martinus Nijhoff, 1986.

(2002). Lecciones de fenomenología de la conciencia interna del tiempo. Madrid: Trotta.

Gallagher, Shaun (1998). The innordinance of Time. Illinois: Northwestern University Press.

McAlister, Linda (1974). "Chisholm and Brentano on Intentionality". The Rewiew of Metaphysics. Vol. 28, No. 2.

Mohanty, Jitendra (2008). The Philosophy of Edmund Husserl. New Haven: Yale University Press: 2011.

_ (2011). Edmund Husserl's Freiburg Years. 1916-1938. New Haven-Londres: Yale University Press.

Mulligan, Kevin y Smith, Barry (1985). "Franz Brentano on the Ontology of Mind". Philosophy and Phenomenological Research. Vol. 45, No. 4.

Enviado: 28/04/2016

Aceptado: 9/07/2016

\section{C)(1)}

ENDOXA está bajo una licencia de Creative Commons Reconocimiento-NoComercial-SinObraDerivada 4.0 Internaciona 
